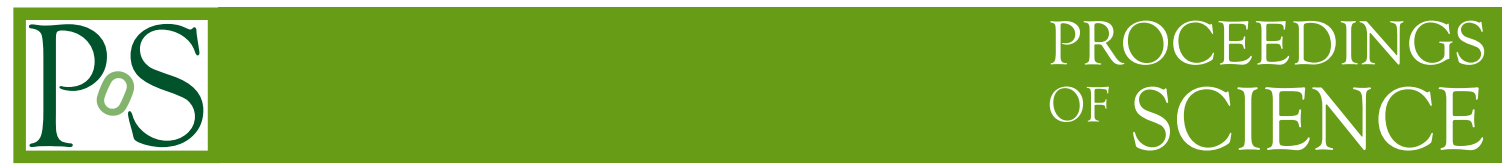

\title{
Strongly Interacting Matter at Very High Energy Density
}

\author{
Larry McLerran** \\ Physics Dept. BNL and RIKEN Brookhaven Center, Upton, NY 11973 USA \\ E-mail: mclerran@bnl.gov
}

I discuss the study of matter at very high energy density. In particular: What are the scientific questions? What are the opportunities to makes significant progress in the study of such matter? What facilities are now or might be available in the future to answer the scientific questions?

XXXIV edition of the Brazilian Workshop on Nuclear Physics,

5-10 June 2011

Foz de IguaÃ ğu, Parana state, Brasil

* Speaker.

${ }^{\dagger}$ A footnote may follow. 


\section{Scientific Questions}

I will focus on the following scientific questions in this talk:

\subsection{What is the nature of thermally equilibrated matter at temperatures and baryon number corresponding to energy density much larger than that of atomic nuclei?}

The study of matter at very high energy density is a natural extension of nuclear physics to scales much larger than those found in atomic nuclei. This matter is found in neutron stars, the formation of black holes, and was present during the big bang. In this talk I will discuss strongly interacting matter, and energy densities $\varepsilon_{\text {Nuclear Matter }}<<\varepsilon<<(100 \mathrm{GeV})^{4}$. (The dynamics of electroweak theory and new particle degrees of freedom will become important when the energy scale is of the order of that of the electroweak scale, and we restrict ourselves to low enough energy density so that only strong and electromagnetic interactions are important.) What are the possible forms of such matter? What are their properties?

\subsection{What are new unexpected forms of matter found in high energy collisions?}

The contribution to a hadron wavefunction that dominates high energy scattering has a very high density of gluons. What is the nature of this matter? Very shortly after the collisions of two high energy nuclei, matter is formed. What are the fundamental properties of such matter and how does it eventually form a thermally equilibrated Quark Gluon Plasma?

\subsection{How are mass and confinement generated?}

How does the presence of matter affect confinement and mass generation? Can we turn off the masses of particles and the effects of confinement? Are there different ways that mass generation can be realized?

\subsection{What is the high energy limit of QCD?}

What is the nature of the interactions of strongly interacting particles at the highest energies? Can we reliably compute particle interactions in this limit?

\section{Thermally Equilibrated Matter}

\subsection{Thermally Equilibrated Matter: Theory}

We know very little, although much is conjectured, about the properties of matter in thermal equilibrium at high energy density. There are a variety of possibilities at finite temperature and baryon number chemical potential. The Quark Gluon Plasma is the fate of QCD at temperatures $T>>\Lambda_{Q C D} \sim 200 \mathrm{MeV}$. This matter is a gas of de-confined quarks and gluons, that at very high temperatures interacts weakly because the interactions of QCD become weak at short distances[1][2]. The linear potential of the vacuum is converted to a Debye screened Coulomb potential. The masses of up and down quarks are very small, much smaller than in the vacuum where their masses are of the order of the QCD scale $\Lambda_{Q C D} \sim 200 \mathrm{MeV}$. The small masses are presumably associated with the evaporation of a condensate of scalar mesons, the chiral condensate, which is responsible 
for mass generation in the vacuum. Chiral symmetry, a symmetry associated with zero mass quarks, is restored.

At high baryon number density, there are a number of proposed phases. Color superconductivity is associated with the condensation of colored Cooper pairs[3]-[4]. Quarkyonic matter can be thought of as a free Fermi gas of quarks with thermal and Fermi surface excitations that are confined[5]. Quarkyonic matter may have novel phases of chiral symmetry breaking[6]-[7]. (It is chiral symmetry breaking that generates the nucleon mass.)

For static properties of the Quark Gluon Plasma, there are high quality computations using lattice gauge theory techniques[8]. Much is understood from theory. Lattice gauge theory however cannot determine the quasi-particle excitations of the system, nor can it be directly applied to heavy ion collisions, where such matter is produced in a laboratory. Experimental tests of the properties of the Quark Gluon Plasma involve hydrodynamic computations which push the limits of numerical computation[9].

Near the cross over between the Quark Gluon Plasma and the Hadronic Matter, it is possible that matter might be very strongly interacting. This has led to methods employing the AdSCFT correspondence. In particular, computations have led to a conjecture concerning a lower bound for the viscosity to entropy ratio[10]. There are also numerous attempts to understand the interactions of high energy probes with the Quark Gluon Plasma[11]. Such computations are qualitative at best however, since the theory for which one extracts the strong coupling limit is not QCD, but a supersymmetric field theory. Also, near the QCD transition, the coupling strength is most probably of order 1, which is close to neither the strong nor the weak coupling limit. Since neither strong nor weak coupling computations provide reliable results, one is forced to consider model computations that presumably mimic much of the correct physics.

For matter at very high density and low temperature, the techniques of lattice gauge theory cannot be used. If one works at baryon chemical potential, $\mu_{B}>>\Lambda_{Q C D}$, there are compelling arguments for the existence of Color Superconductivity. Color Superconductivity is the formation of bound states of quarks near the Fermi surface, colored Cooper pairs. These colored Cooper pairs form condensates. There is a rich phenomenology of such matter, and it may be important for the cores of neutron stars. For the baryon number densities and temperatures typical of heavy ion collisions, it is not yet possible to do first principle computations, and one relies on model computations with much uncertainty. Conclusions drawn from such models are qualitative to semiquantitative, at best.

Quarkyonic Matter was discovered in studies of QCD at large baryon number density in the large $N_{c}$ limit of QCD, In this limit baryons are very massive, $M_{B} \sim N_{c} \Lambda_{Q C D}$, and the baryon number density itself becomes an order parameter for deconfinement. Ordinary hadronic matter contains no baryons because the baryons are so heavy. In the deconfined phase there can be baryons, because baryon number appears in quarks, and quarks have masses of order $\Lambda_{Q C D}$. There is a third phase, Quarkyonic, which is confined and has baryons present. The baryons are forced on the system if the baryon number chemical potential $\mu_{B} \geq M_{B}$. Confinement persists in the presence of a very high density of baryons because in the large $N_{c}$ limit, quarks do not affect the confining potential. It turns out that chiral symmetry remains broken in Quarkyonic Matter, but the chiral condensate is crystalline and composed of chiral spirals (configurations of scalar particle condensates which rotate back and forth into vector mesons). One can think of Quarkyonic Matter 


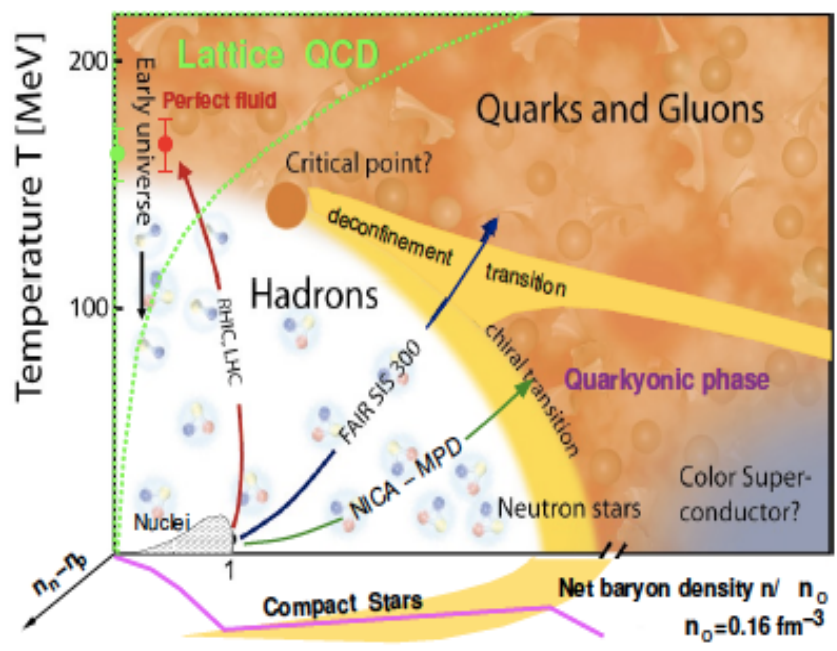

Figure 1: A hypothetical phase diagram of QCD

as a Fermi sea of free massless quarks surrounded by a Fermi surfaces that is both confining and chiral symmetry breaking. Thermal and Fermi surface excitations are confined. The dynamics of the Fermi sea is that of effectively free massless quarks because the typical Fermi energy can be $\mu_{\text {quark }}>>\Lambda_{Q C D}$. For finite $N_{c}$, it is difficult to do computations and little is known from first principle. It could be that there is a cross over from Hadronic Matter to Quarkyonic or there might be a true phase transition. Quarkyonic Matter, if it exists, should begin to appear when matter is compressed to little more than the density of nuclear matter, and as such will be relevant for the properties of neutron star interiors. It might be probed in low to intermediate energy heavy ion collisions.

A hypothetical phase diagram of QCD is shown in Fig. 1. There are two distinctive features of this diagram: There is a triple point, where the Quarkyonic, Hadronic and Quark Gluon Plasma phases meet. There is also a critical point where the assumed first order phase transition line that separates the Hadronic world from the Quarkyonic world, and extending into the HadronicQGP boundary, ends. It is known from lattice Monte-Carlo computation that the phase transitions separating the Hadronic world from the QGP is a cross over at low value of $\mu_{B}$. Whether or not there are first order transitions in the phase diagram, and if there is, where is the critical point, are all theoretical conjecture at this point. There is some evidence from low energy heavy ion experiments for the existence of the Hadron-Quarkyonic transition and for a triple point[12]. The study of this phase diagram is the subject of experiments at RHIC, and proposed experiments at FAIR and NICA accelerators[13]-[14].

\subsection{Thermally Equilibrated Matter: Experiment}

Two major accelerator facilities have ongoing major efforts dedicated to the study of the Quark 
Gluon Plasma using ultra-relativistic heavy ion collisions. These are successors to the earlier fixed target experiments at the AGS (Brookhaven National Laboratory), and at the CERN-SPS. The CERN SPS announced that they had seen hints of the existence of a Quark Gluon Plasma. The RHIC experiments have provided compelling evidence for the existence of a Quark Gluon Plasma. The early results from the LHC have largely confirmed the observations of RHIC, and produced surprising results concerning the opacity of the Quark Gluon Plasma to highly energetic probes.

Measurements of the fluid properties of this produced matter and its opacity to energetic particles have led to the conclusion that this matter is strongly self-interacting. The remarkable agreement between hydrodynamic computations of the properties of matter produced in these collisions and experimental measurement have further led to the hypothesis that the Quark Gluon Plasma is a nearly perfect fluid.

The Relativistic Heavy Ion Collider (RHIC) at Brookhaven National Laboratory can produce colliding beams of nuclei up to an energy of $100 \mathrm{GeV} / \mathrm{Nucleon}$. RHIC is dedicated to the study of nucleus-nucleus interactions, and collisions of polarized protons. There were originally four heavy ion experiments. Brahm's provided early measurements on rapidity and transverse momenta of various species of particles. (It provided the first hints for the Color Glass Condensate at RHIC, which will be discussed later.) Phobos provided the first measurement of particle multiplicities, studied global properties of heavy ion collisions, and has provided much information about correlations between particle mutlplicity and centrality of collisions. Phenix is a large experiment with about 450 experimenters from 11 countries that has made discoveries associated with jet quenching (opacity of highly energetic probes), electromagnetic particle production, and flow. The STAR experiment is a general purpose detector with 500 experimenters from 12 counties. It has made discoveries associated with jet quenching, flow, and anti-matter production.

The LHC accelerates protons and heavy ions up to several $\mathrm{TeV} / \mathrm{Nucleon}$. It has one dedicated heavy ion experiment with about 1000 experimenters from 33 countries. Alice can measure and identify particles produced at low transverse momentum. There are efforts on heavy ions embedded within the CMS and Atlas experiments. CMS can provide global event information over a large range in rapidity. Both CMS and Atlas are well instrumented for studying high transverse momentum jets.

The major early experimental results from RHIC are summarized in Refs.[16]-[18]. These include opacity of the quark matter to high energy probes or jet quenching, and the fluid behaviour of such matter, or flow.

In high energy hadron-hadron collisions, high transverse momentum particles, jets, are produced. If there is a media present, these jets may scatter from the media and loose some of their transverse momentum. This was observed to be the case in the RHIC experiment. The degree of jet quenching was much larger than expected, and is still not fully understood from theory. In Fig. 2, the ratio of produced jets to that expected without media interaction is shown. The upper curve is for peripheral collisions where not much matter is present. The lower curve is for higher centrality.

The results on jet quenching have been verified and much extended by the LHC experiments. Both the Atlas and CMS experiments [19]-[20] can measure jet quenching, and the deposition of energy of particles along the direction of the jet (jet fragmentation function). Results from CMS are shown in Fig. 3. The energy loss seems to be dominated by the modification of particles with low 


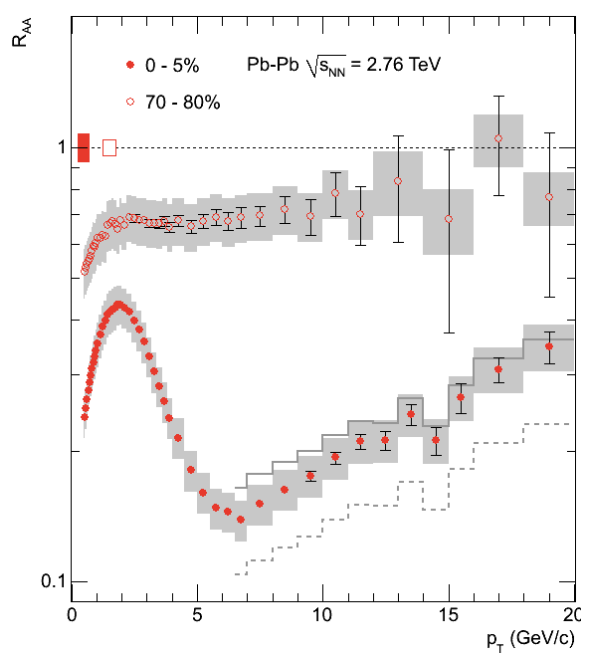

Figure 2: Jet quenching at RHIC

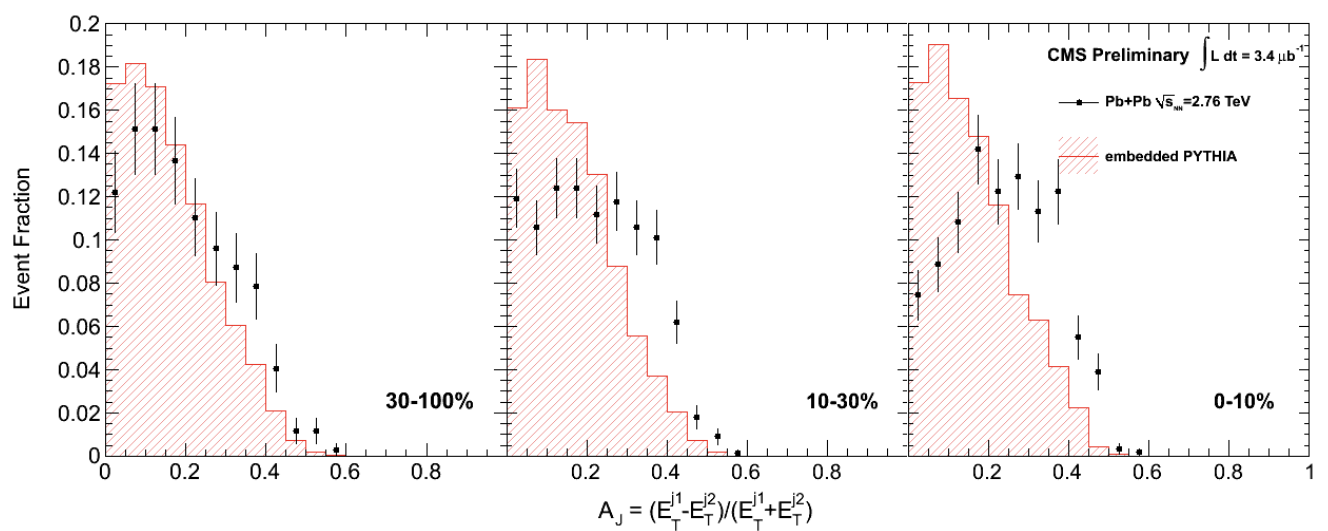

Figure 3: Energy loss along the jet axis at CMS

momentum along the jet axis, qualitatively in accord with early models based on field theory[21].

One of the first measurements done at RHIC was to determine the degree to which the matter produced flows like a fluid. If the matter has fluid properties, then in off center collisions,, matter should collectively flow in the reaction plane of the collision. The magnitude of this flow is predicted by hydrodynamic computations, and is quantified in a variable $v_{2}$, which measures the angular asymmetry induced by flow. Results of a STAR measurement of such flow for various centralities is shown in Fig. [22]. these flow computations agree well with hydrodynamic model computations involving small viscosity (small mean free paths for particle interactions). Similar strong flow effects have been very clearly demonstrated in the Alice experiment at the LHC[23].

The experimental measurements of jet quenching have led to the conclusion that the Quark Gluon Plasma produced at RHIC is strongly interacting. The agreement with fluid dynamic simulations for flow and various particle distributions have led to the conjecture that the matter produced 


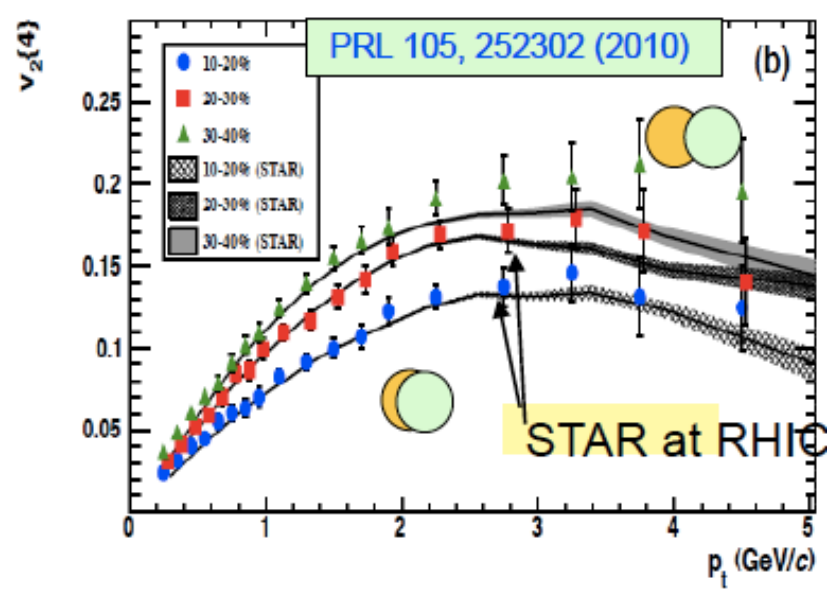

Figure 4: Flow as a function of centrailty in the STAR experiment

has small ratio of viscosity to entropy, and the strongly interacting Quark Gluon Plasma, or sQGP paradigm. In both these cases, the theoretical understanding is qualitative and semi-quantitative. In the jet quenching case, interactions take place over all times in the collisions, and one needs to account for the properties of an increasingly complicated media. For theoretical descriptions both jet quenching and flow, results are also sensitive to the physics at early times when the system is not yet a thermalized Quark Gluon Plasma.

The issue of how hot the sQGP might have been has been addressed in photon and dilepton measurements from the Phenix Collaboration[24]. Photon and dileptons are interesting probes since their small electromagnetic interaction cross sections let them escape the produced matter with little interaction. An excess of photons has been measured in the range of $1 \mathrm{GeV} \leq p_{T} \leq$ $3 \mathrm{GeV}$ for central collisions, as shown in Fig. 5. Similarly there is an excess in the yield of dileptons for dilepton masses $200 \mathrm{MeV} \leq M \leq 1 \mathrm{GeV}$. The magnitude and shape of the photon yield can be fit by invoking the QGP hypothesis and using hydrodynamic computations. The yield of dileptons has a similar excess, although it has proven not possible to fit the excess using a QGP hypothesis. The photons apparently flow with produced mater, and the dileptons have an anomalously small transverse momenta. Both these features have not been possible to describe within the conventional QGP hypothesis. These results present a mystery and suggest to me that the underlying physics, which is the conventional thermalized QGP hypothesis plus hydrodynamics, may need some fundamental revision. The properties of the matter produced at RHIC suggest a very strongly interacting fluid. The degrees of freedom at the energy density scales probed are most certainly those of quarks and gluons. But do they arise from a thermally equilibrated quark gluon plasma, or is the physics more subtle?

There is the possibility to study anomalous properties of strongly interacting gauge theories[25][26]. Anomalous processes are associated with topological charge fluctuations, and can induce event by event parity violation. Analogous effects in electroweak theory might ultimately be responsible for generating the baryon asymmetry of the universe. Such effects might be seen in correlations in charge particle production with the reaction plane[27]. There are possible background 


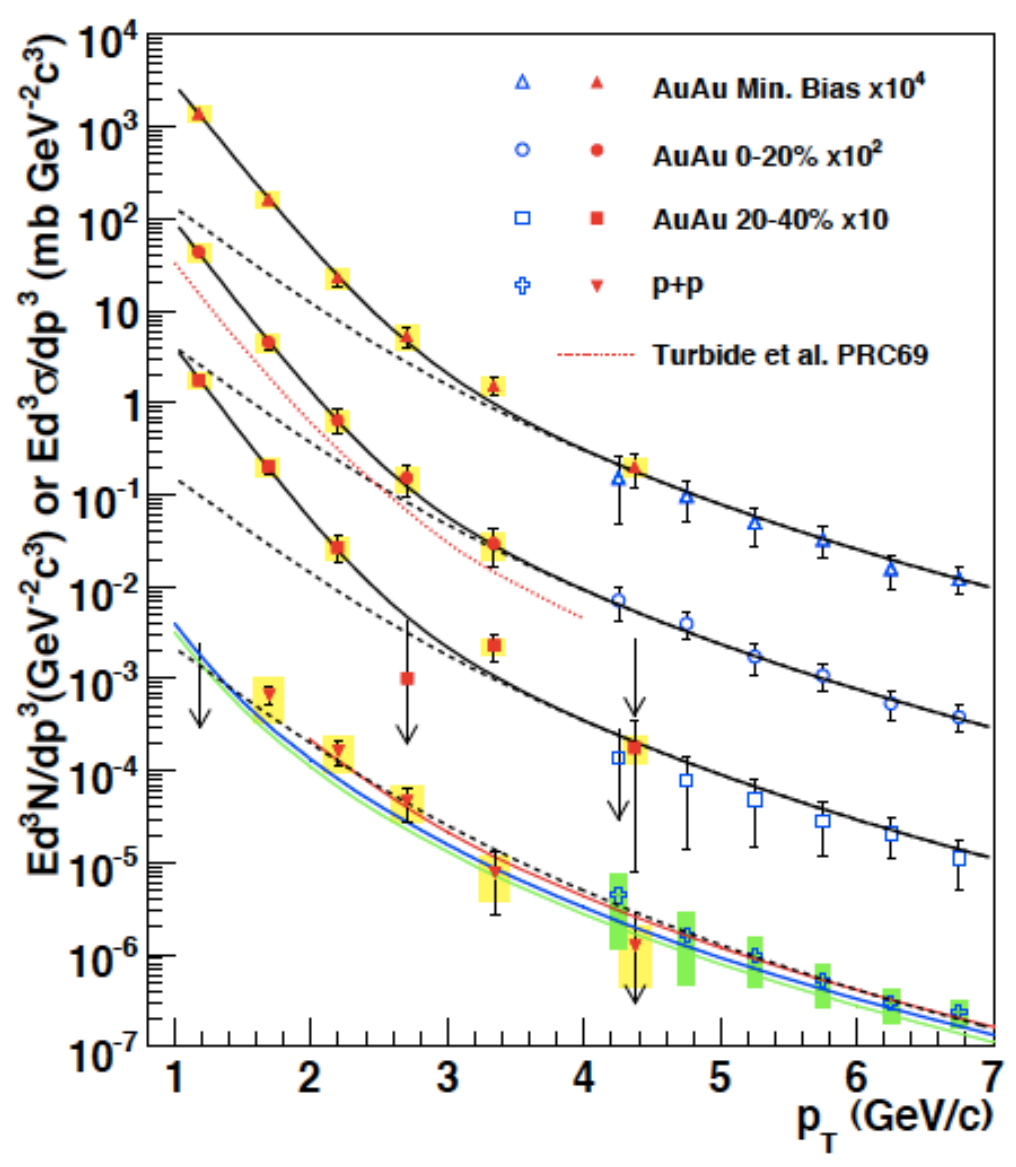

Figure 5: The excess of photon measured as a function of centrality in the Phenix experiment.

effects that might fake proposed signal that need careful examination.

To study matter at very high baryon number density and low to intermediate temperatures, one needs collisions at center of mass energies of $30 \mathrm{GeV}$ and lower. There were previous fixed target experiments done at the CERN-SPS and the AGS at Brookhaven National Laboratory. In hindsight, I believe these experiments provided hints of the formation of a Quark Gluon Plasma[28]. The RHIC accelerators has been run in a low energy mode, $E / A \sim 10 \mathrm{GeV}$, which provided data using central detectors in the center of mass frame. There are proposals for the Compressed Baryonic Matter experiment at FAIR, and for a central detector at NICA in Dubna. This area of research, both theoretical and experimental, should be carefully thought through again in light of both theoretical developments on the properties of high density baryonic matter, recent results from lattice gauge theory[29], and experimental discoveries at RHIC and LHC. 

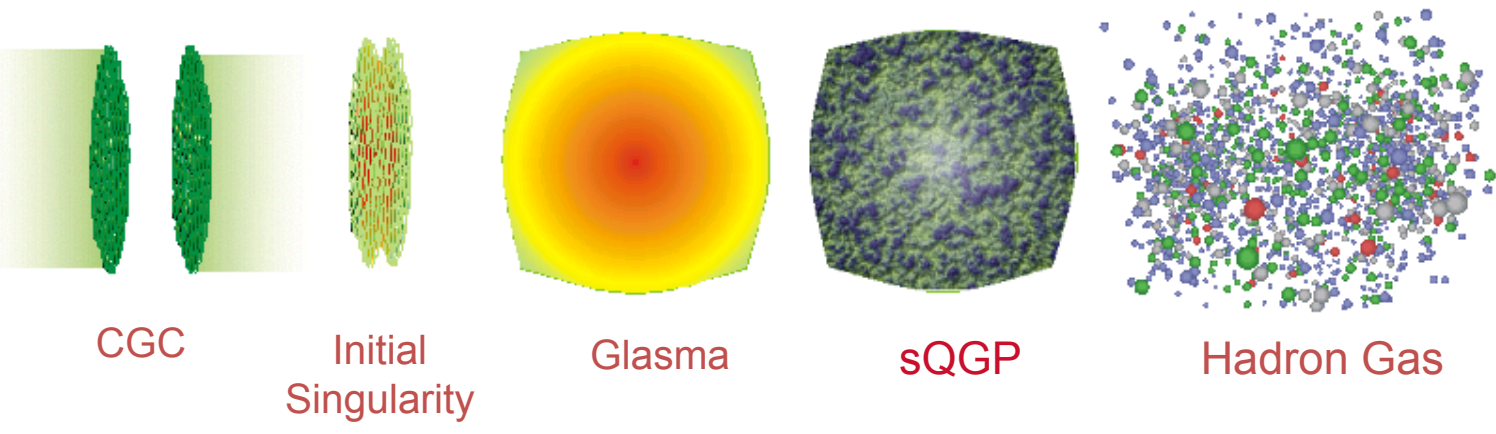

Singularity

Hadron Gas

Figure 6: The various stages in the evolution of a heavy ion collision.

\section{What are unexpected new forms of matter produced in heavy ion collisions?}

\subsection{Unexpected new forms of matter: Theory}

A figure showing the "Standard Model" of high energy nucleus nucleus collisions in shown in Fig. 6. The initial nuclei are composed of a high density of gluons. These gluons form a new state of matter, the Color Glass Condensate. As the sheets of CGC pass through one another, they acquire local color electric and color magnetic charge density, which is equal in magnitude but opposite in sign on the two sheets of CGC. This color charge density induces longitudinal color electric and color magnetic fields. This is the initial state of the Glasma. The Glasma first evolves largely by classical time evolution decaying into gluons. These gluons eventually thermalize forming a strongly interacting Quark Gluon Plasma. This sQGP in turn ultimately forms a Hadron Gas, that itself eventually decouples into free mesons and baryon that propagate to detectors.

In this picture, two new forms of matter appear: the CGC and the Glasma. The current status of our understanding of the CGC is reviewed in Ref. [30]. The CGC is best conceptualized by thinking about a hadron in fast moving frame. A constituent gluon has some fractional momentum of the hadron. If one measures the number of gluons at small $\mathrm{x}$, they increase without bound, as is shown in Fig. 7. The gluons totally dominate the hadron wavefunction by $x \sim 10^{-1}-10^{-2}$.

At small $\mathrm{x}$, the gluon occupation number becomes very large. The strong coupling is weak because the density of gluons is very large[31]-[32]. The gluons can be described as a highly coherent classical field[33]-[33]. This is a highly Lorentz boosted Coulombic field, which under such boost become transverse color electric and color magnetic fields.

When two sheets of CGC collide, the color fields of the two CGC's interact. This process may be treated classically[35]. In the collision, the sheets of CGC acquire a local color electric and color magnetic charge density and lines of longitudinal color electric and color magnetic field join them . These fields evolve and decay locally producing gluons [36]-[37].

As the system evolves, the gluons may thermalize. Exactly how this happens is not known. There are conjectures that the Glasma may strongly interact with itself due to coherence of the classical fields and there may be a Bose condensation of gluons[38]-[39]. The system might also decouple for a while, until the gluons are far enough separated for strong coupling effects to thermalize the system[40]. 


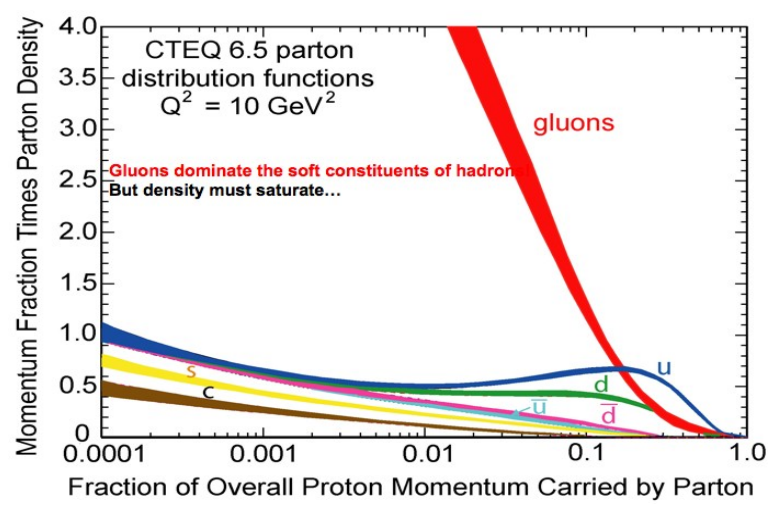

Figure 7: The gluon number at small $\mathrm{x}$. .

The theory of the CGC and Glasma are sound in the limit of extremely high energies where the coupling is very weak. At RHIC energies, and to a lesser degree at the LHC, such an approximation is questionable. Many computations invoke model hypothesis since either the variable computed is sensitive to physics for which there is not control, or well motivated first principle computations have not yet been done.

\section{Unexpected new forms of matter: Experiment}

The earliest experimental hints of the existence of the Color Glass Condensate came from scaling properties of deep inelastic scattering experiments[41]. Early in the RHIC experiments, the observed scaling of the multiplicity of particles with centrality gave results consistent with a CGC hypothesis. Much excitement was generated by the Brahms result which showed a suppression of hard particle production with multiplicity, rapidity and energy that followed predictions for the CGC[17]. Computation of these effects from first principles is an area of improving quality at this time, but needs work to make it precise and better reliable[42].

Perhaps the most direct result to date comes from the measurement of high $p_{T}$ particle suppression seen in $p A$ collisions. By observing the disappearance of forward-backward azimuthal angular correlation in $d A$ collision in such collisions, one can study the quenching of a high $p_{T}$ particle produced in association with a trigger particle. By requiring that the two particles have momentum close to the deuteron, one can exclude nuclear target fragmentation effects, and enhance the small $\mathrm{x}$ contribution of the nuclear wavefunction. A study of such correlations has been done by Star and Phenix[43]. There is a disappearance of the backward peak in central dAu collisions that has been interpreted as "jet quenching" due to coherent scattering on the CGC. This is shown in Fig. 8. Such studies would be greatly improved by $p P b$ measurements at LHC energies, where the $x$ values of the nucleus are very small. Theoretical computations are approaching reliable first principle computations of these effects[44]-[47].

The Glasma flux tubes generate long range correlations in rapidity. The simplest of such correlations is that the central particle multiplicity is strongly correlated with the number of nucleon participants in a heavy ion collision. One can measure correlations between particle multiplicity 


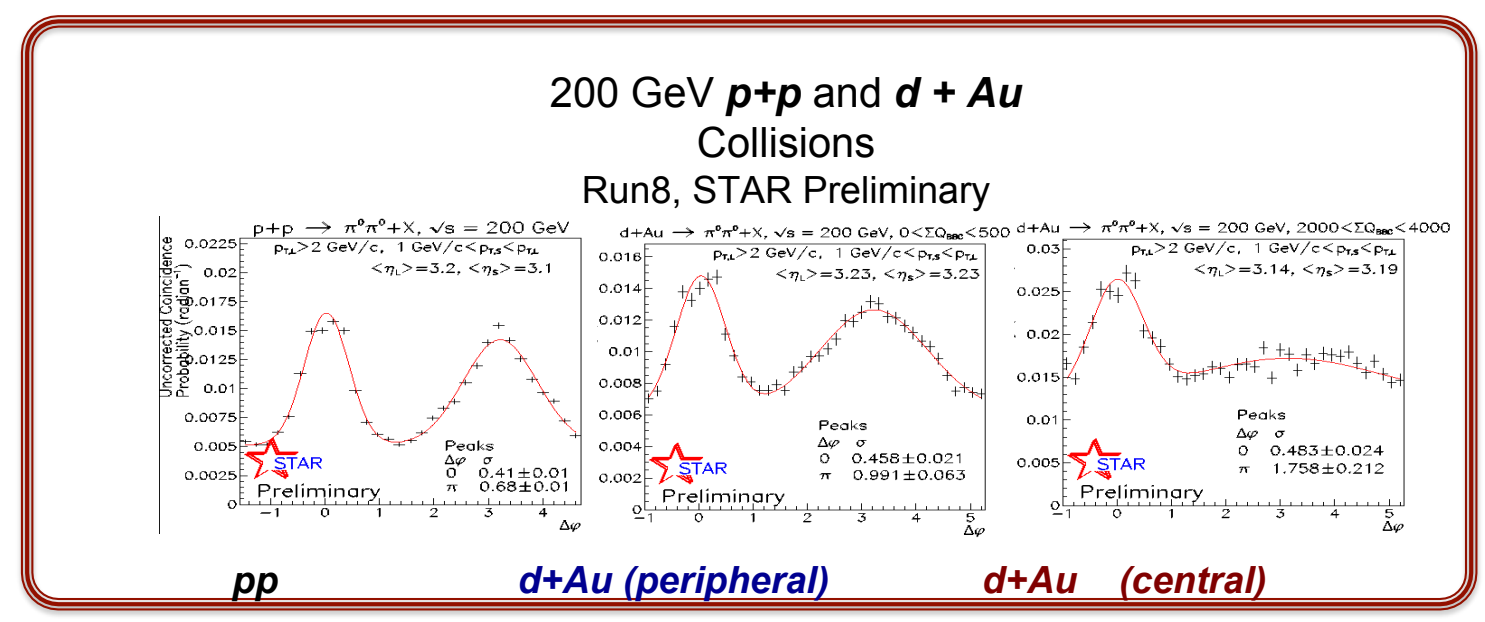

Figure 8: Forward backward angular correlations in dAu and pp collisions

in various rapidity bins, and one finds very strong correlations, in fact correlations that increase in strength as the centrality of the collision is increased[48], and become very large..

In general, Glasma flux tube are tied to sources, and fluctuations in the positions of these sources in the fragmentation region will result in corresponding local fluctuations in the central region[49]. These correlations will be long range in rapidity, and when plotted as a function of angle and rapidity will generate a ridge structure. Such correlations were seen in the hydrodynamic simulations of the Brazil group[9], and in the computations of Ref. [50]. One should see such correlations in various flow measurements, and there is now an entire framework for relating various asymmetries in the flow distribution initial state fluctuations[51]. One might further question whether or not the fluctuations of sources that produce these flow patterns are fluctuations in nucleon position only, or whether they might include fluctuations in color charge sources at the subatomic scale. The answer is provided by the results from the CMS collaboration that show that there exist long range correlations of the type predicted by Glasma flux tubes in fluctuation in the pp experimental data[52]- [53]. Such a correlation is shown in Fig. 9. This result also suggests that some of the two particle correlation that produces the ridge might have a non-flow origin. The issue of computing and measuring high order moments of the flow distribution is a fascinating problem, and may provide information about initial conditions similar to the moments of the black body spectrum in cosmology[54].

The photon and dilepton results seen in Phenix may have their origin in the Glasma. The Glasma will surely produce particles in the kinematic range seen by Phenix, and it should be possible to compute distributions. Such distributions possess the property of scaling in powers of the density of gluons divided by a typical momentum scale squared (geometrical scaling). It might be possible to explain the dominance of low $p_{T}$ dileptons in the dilepton excess if there is a Bose condensate of gluons present. One needs much more experimental data to properly test these ideas. Such results must also be confirmed in the LHC experiments.

A surprise of the LHC experiments was how well saturation concepts work for describing 

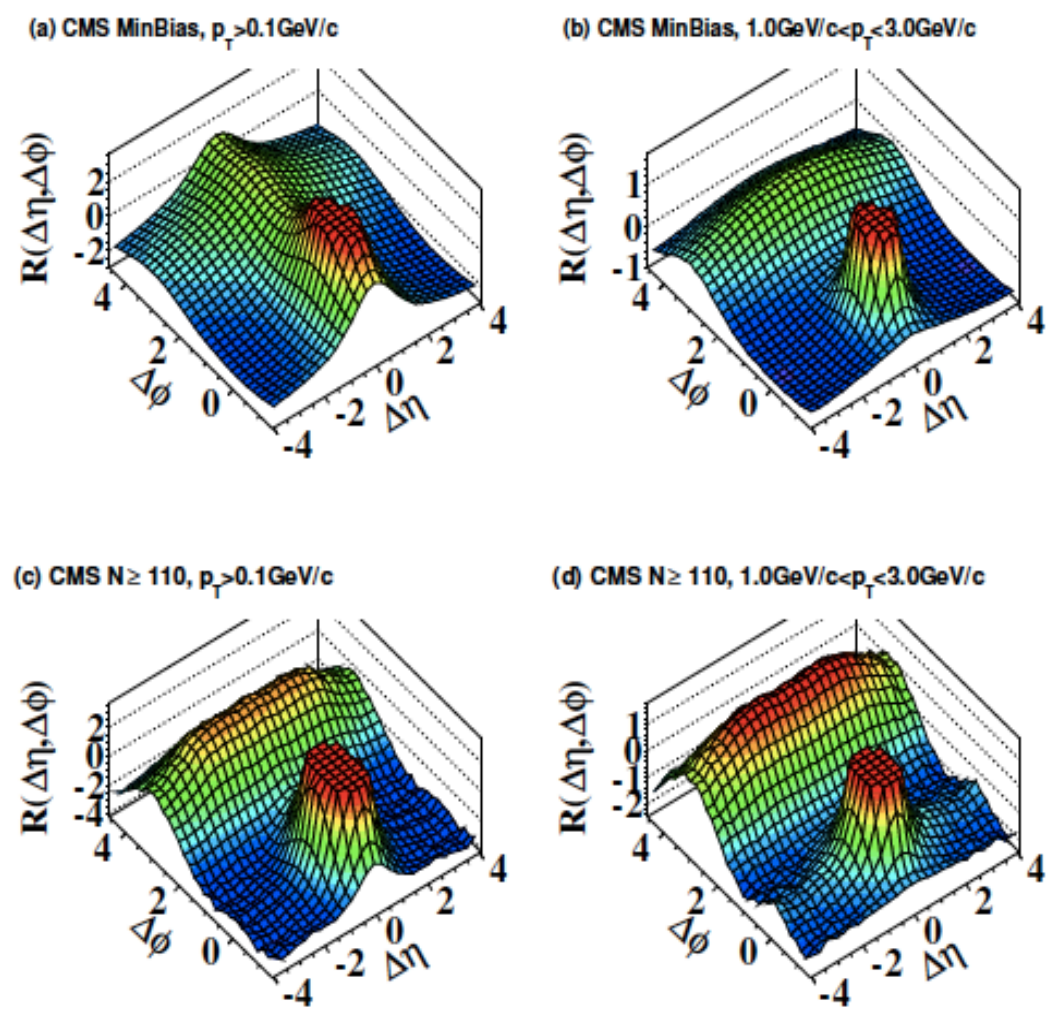

Figure 9: The ridge as seen in the CMS experiment at LHC.

scaling properties, correlation between transverse momentum and multiplicity, and multiplicity fluctuations in the LHC pp data [55]-[56]. The implications of these results for nucleus-nucleus collisions at LHC energies are largely unexplored.

One of the future directions for testing ideas associated with the Color Glass Condensate and gluon saturation involve electron-ion collisions. There are proposals for a polarized ep and eA collider either at Brookhaven National Laboratory or at Thomas Jefferson National Laboratory. There are groups actively working on possible experimental setups[57].

A possible future direction at $\mathrm{LHC}$ is the study $\mathrm{pPb}$ collisions. This will provide data on the gluon and quark distributions in nuclei at very small $\mathrm{x}$, and is unique in the $\mathrm{x}$ range probed. In any case, such experiments will very likely be necessary to sort out various effects as uniquely due to the Quark Gluon Plasma and Glasma vs that of the Color Glass Condensate.

\section{Summary}

The theoretical and experimental study of new forms of high energy density matter is still very much a "wild west" field. There is much freedom for developing new concepts which can have order one effects on the way we think about such matter. It is also a largely "lawless" field, in that concepts and methods are being developed as new information is generated. There is also great 
possibility for new experimental discovery. Most of the exciting results from RHIC experiments were unanticipated. The methods used for studying various effects like flow, jet quenching, the ridge, two particle correlations etc. were developed as experiments evolved. I believe this will continue to be the case at LHC and as we use existing and proposed accelerators to turn theoretical conjecture into tangible reality. At some point this will no doubt evolve into a precision science, and that will make the field more respectable, but for my taste, the "wild west" times are the most fun.

\section{Acknowledgements}

I gratefully acknowledge the support of the Brazilian Physical Society, where the contents of this paper were presented. I am supported under DOE Contract No. DE-AC02-98CH10886. I acknowledge the Heidelberg Theoretical Physics Institute and the Hans Jensen Prize for supporting my research while this manuscript was completed..

\section{References}

[1] N. Cabibbo, G. Parisi, Phys. Lett. B59, $67-69$ (1975).

[2] J. C. Collins, M. J. Perry, Phys. Rev. Lett. 34, 1353 (1975).

[3] R. Rapp, T. Schafer, E. V. Shuryak, M. Velkovsky, Phys. Rev. Lett. 81, 53-56 (1998). [hep-ph/9711396].

[4] M. G. Alford, K. Rajagopal, F. Wilczek, Phys. Lett. B422, 247-256 (1998). [hep-ph/9711395].

[5] L. McLerran, R. D. Pisarski, Nucl. Phys. A796, 83-100 (2007). [arXiv:0706.2191 [hep-ph]].

[6] L. Y. .Glozman, R. F. Wagenbrunn, Phys. Rev. D77, 054027 (2008). [arXiv:0709.3080 [hep-ph]].

[7] T. Kojo, Y. Hidaka, L. McLerran, R. D. Pisarski, Nucl. Phys. A843, $37-58$ (2010). [arXiv:0912.3800 [hep-ph]].

[8] F. Karsch, Lect. Notes Phys. 583, 209-249 (2002). [hep-lat/0106019].

[9] Y. Hama, T. Kodama, O. Socolowski, Jr., Braz. J. Phys. 35, 24-51 (2005). [hep-ph/0407264].

[10] P. Kovtun, D. T. Son, A. O. Starinets, Phys. Rev. Lett. 94, 111601 (2005). [hep-th/0405231].

[11] H. Liu, K. Rajagopal, U. A. Wiedemann, Phys. Rev. Lett. 97, 182301 (2006). [hep-ph/0605178].

[12] A. Andronic, D. Blaschke, P. Braun-Munzinger, J. Cleymans, K. Fukushima, L. D. McLerran, H. Oeschler, R. D. Pisarski et al., Nucl. Phys. A837, 65-86 (2010). [arXiv:0911.4806 [hep-ph]].

[13] P. Senger, Nucl. Phys. A862-863, 139-145 (2011).

[14] A. Sorin, V. Kekelidze, A. Kovalenko, R. Lednicky, I. Meshkov, G. Trubnikov, Nucl. Phys. A855, 510-513 (2011).

[15] I. Arsene et al. [ BRAHMS Collaboration ], Nucl. Phys. A757, 1-27 (2005). [nucl-ex/0410020].

[16] B. B. Back, M. D. Baker, M. Ballintijn, D. S. Barton, B. Becker, R. R. Betts, A. A. Bickley, R. Bindel et al., Nucl. Phys. A757, 28-101 (2005). [nucl-ex/0410022].

[17] I. Arsene et al. [ BRAHMS Collaboration ], Nucl. Phys. A757, 1-27 (2005). [nucl-ex/0410020]. 
[18] K. Adcox et al. [ PHENIX Collaboration ], Nucl. Phys. A757, 184-283 (2005). [nucl-ex/0410003].

[19] S. Chatrchyan et al. [ CMS Collaboration ], Phys. Rev. C84, 024906 (2011). [arXiv:1102.1957 [nucl-ex]].

[20] G. Aad et al. [ ATLAS Collaboration ], Phys. Rev. Lett. 105, 161801 (2010). [arXiv:1008.2461 [hep-ex]].

[21] J. Casalderrey-Solana, J. G. Milhano, U. A. Wiedemann, J. Phys. G G38, 035006 (2011). [arXiv:1012.0745 [hep-ph]].

[22] B. I. Abelev et al. [ STAR Collaboration ], Phys. Rev. Lett. 101, 252301 (2008). [arXiv:0807.1518 [nucl-ex]].

[23] KAamodt et al. [ The ALICE Collaboration ], Phys. Rev. Lett. 105, 252302 (2010). [arXiv:1011.3914 [nucl-ex]].

[24] A. Adare et al. [ PHENIX Collaboration ], Phys. Rev. C81, 034911 (2010). [arXiv:0912.0244 [nucl-ex]].

[25] D. Kharzeev, R. D. Pisarski, M. H. G. Tytgat, Phys. Rev. Lett. 81, 512-515 (1998). [hep-ph/9804221].

[26] D. E. Kharzeev, L. D. McLerran, H. J. Warringa, Nucl. Phys. A803, 227-253 (2008). [arXiv:0711.0950 [hep-ph]].

[27] B. I. Abelev et al. [STAR Collaboration], Phys. Rev. C 81, 054908 (2010) [arXiv:0909.1717 [nucl-ex]].

[28] U. W. Heinz, M. Jacob, [nucl-th/0002042].

[29] F. Karsch, K. Redlich, Phys. Lett. B695, 136-142 (2011). [arXiv:1007.2581 [hep-ph]].

[30] J. P. Blaizot, Nucl. Phys. A854, 237-256 (2011). [arXiv:1101.0260 [hep-ph]].

[31] L. V. Gribov, E. M. Levin, M. G. Ryskin, Phys. Rept. 100, 1-150 (1983).

[32] A. H. Mueller, J. -w. Qiu, Nucl. Phys. B268, 427 (1986).

[33] L. D. McLerran, R. Venugopalan, Phys. Rev. D49, 2233-2241 (1994). [arXiv:hep-ph/9309289 [hep-ph]].

[34] L. D. McLerran, R. Venugopalan, Phys. Rev. D49, 3352-3355 (1994). [hep-ph/9311205].

[35] A. Kovner, L. D. McLerran, H. Weigert, Phys. Rev. D52, 6231-6237 (1995). [hep-ph/9502289].

[36] A. Krasnitz, R. Venugopalan, Phys. Rev. Lett. 84, 4309-4312 (2000). [hep-ph/9909203].

[37] T. Lappi, Phys. Rev. C67, 054903 (2003). [hep-ph/0303076].

[38] R. Baier, A. H. Mueller, D. Schiff, D. T. Son, Phys. Lett. B502, $51-58$ (2001). [arXiv:hep-ph/0009237 [hep-ph]].

[39] J. -P. Blaizot, F. Gelis, J. Liao, L. McLerran, R. Venugopalan, [arXiv:1107.5296 [hep-ph]

[40] R. A. Janik, R. B. Peschanski, Phys. Rev. D73, 045013 (2006). [hep-th/0512162].

[41] L. McLerran, (ed.), J. Dunlop, (ed.), D. Morrison, (ed.), R. Venugopalan, (ed.), Nucl. Phys. A854, $1-256(2011)$

[42] J. L. Albacete, A. Dumitru, Y. Nara, [arXiv:1106.0978 [nucl-th]].

[43] E. Braidot for the STAR collaboration, arXiv:1005.2378; B. A. Meredeth for the Phenic Collaboration, PoS DIS 2010, 081 (2010). 
[44] C. Marquet, [arXiv:1101.3466 [hep-ph]].

[45] J. L. Albacete, C. Marquet, Phys. Rev. Lett. 105, 162301 (2010). [arXiv:1005.4065 [hep-ph]].

[46] K. Tuchin, Nucl. Phys. A855, 502-505 (2011). [arXiv:1012.4709 [hep-ph]].

[47] B. -W. Xiao, F. Yuan, Nucl. Phys. A854, 180-186 (2011).

[48] B. I. Abelev et al. [ STAR Collaboration ], Phys. Rev. Lett. 103, 172301 (2009). [arXiv:0905.0237 [nucl-ex]].

[49] J. Adams et al. [ STAR Collaboration ], Phys. Rev. Lett. 93, 252301 (2004). [nucl-ex/0407007].

[50] K. Werner, T. Hirano, I. .Karpenko, T. Pierog, S. Porteboeuf, M. Bleicher, S. Haussler, Nucl. Phys. Proc. Suppl. 196, 36-43 (2009).

[51] B. Alver, G. Roland, Phys. Rev. C81, 054905 (2010). [arXiv:1003.0194 [nucl-th]].

[52] V. Khachatryan et al. [ CMS Collaboration ], JHEP 1009, 091 (2010). [arXiv:1009.4122 [hep-ex]].

[53] A. Dumitru, K. Dusling, F. Gelis, J. Jalilian-Marian, T. Lappi, R. Venugopalan, Phys. Lett. B697, 21-25 (2011). [arXiv:1009.5295 [hep-ph]].

[54] P. Staig, E. Shuryak, [arXiv:1106.3243 [hep-ph]].

[55] L. McLerran, M. Praszalowicz, Acta Phys. Polon. B41, 1917-1926 (2010). [arXiv:1006.4293 [hep-ph]].

[56] P. Tribedy, R. Venugopalan, Nucl. Phys. A850, 136-156 (2011). [arXiv:1011.1895 [hep-ph]].

[57] D. Boer, M. Diehl, R. Milner, R. Venugopalan, W. Vogelsang, D. Kaplan, H. Montgomery, S. Vigdor et al., [arXiv:1108.1713 [nucl-th]]. 